\title{
Abstracts of papers presented at the Mammalian Genetics Group meeting held in the Linnean Society Rooms, Piccadilly, London on 22 and 23 November 1983
}

\section{Evolution of nuclear and mitochondrial genomes in European wild mice}

\author{
By F. BONHOMME, P. BOURSOT, Ph. FORT and L. THALER \\ Institut des Sciences de l'Evolution-Laboratoire de Génétique, \\ Université des Sciences et Techniques du Languedoc, \\ Place Eugène Bataillon-34060-Montpellier Cedex
}

The biogeography of the Mus musculus complex of species in Europe is now well known. Five biochemical groups interact in different ways, and protein electrophoresis has permitted to evaluate the genetic exchanges between the five groups, as well as to reconstruct their phylogeny. The same is now available for mt DNA through endonuclease restriction analysis and direct sequencing. The patterns obtained for mitochondrial and nuclear genomes are not always concordant. Processes of speciation are discussed under this light, a special stress being put in this presentation on the groups Mus 1 and Mus 2, which interact along a hybrid zone running throughout central Europe from Denmark to the Black Sea.

\section{Mouse oocyte vs. spermatocyte meiosis: pachytene analysis in trisomy 19}

\author{
BY A. GROPP, R. JOHANNISSON AND H. WINKING \\ Institut für Pathologie, Medizinische Hochschule Lübeck, Ratzeburger Allee 160, \\ 2400 Lübeck
}

The chromosomal pairing behaviour in meiotic prophase was studied in murine Ts 19 using fetal oocytes and spermatocytes from young males (age 18 and 27 days). Ts 19 had been induced on a chromosomal background with heterozygosity for two partially homologous Robertsonian chromosomes which, in a balanced condition, form a quadrivalent. In oocytes as well as in spermatocytes synaptonemal pairing complexes were found either (rarely) with a quadrivalent plus a univalent acrocentric no. 19 in its proximity or (frequently) with incomplete pentavalent formation showing triplex pairing of the distal segments of the three chromosomes 19, occasional full length pairing of the acrocentric no. 19 with the Rb no. 19 arms or casual non-homologous association of the proximal segment of the acrocentric. A further unexpected finding was triradial pairing of the no. 19 acrocentric with 
the straddled distal segments of the no. 19 arms of both Rb chromosomes. Such association is, by the nature of this pairing pattern, partially non-homologous. A special feature of male pachytene was a frequent spatial association of the multivalent with the $X Y$-sex chromosomal bivalent. (Supported by Deutsche Forschungsgemeinschaft.)

\title{
Oocytes in XY sex reversed and hermaphroditic mice
}

\author{
By A. GROPP AND H. WINKING \\ Institut für Pathologie der Medizinischen Hochschule Lübeck, D-2400 Lübeck, \\ West Germany
}

The phenomenon of partial or complete sex reversal among the $X Y$ progeny of C57BL/6 mice possessing a 'foreign' $Y$-chromosome, was used (1) to study directly the behaviour of $X$ and $Y$ chromosomes in fetal oocytes, and (2) to follow the fate of $X Y$ germ cells during ovarian and non-ovarian development of the gonads. In meiotic prophase of fetal oocyte-type $X Y$-germ cells from ovary-like gonads $X$ and $Y$-univalency was frequent, probably resulting from precocious separation. Both chromosomes showed often loops caused by segmental self-pairing. The absence of a sex vesicle suggests sex chromosome activity, at least of the $X$. Histologically, massive degeneration of oocytes is observed in the ovary-like gonads during late fetal development, so that only a reduced number of primary, secondary, and Graafian follicles is present in the ovary of young adult $X Y$ females. Atresia at all stages of follicular maturation, and formation of interstitial gland masses from theca interna cells are prominent features of the $X Y$ ovary, which is of smaller size than normal. In young adult hermaphrodites large oocytes with zona pellucida, but almost devoid of follicular cells, are often observed in testicular-type tubules of the gonads. They can remain as shadow casts if, with increasing age of the $X Y$ individual, the oocytes degenerate. (Supported by Deutsche Forschungsgemeinschaft.)

\section{Interest of interspecific mouse hybrids for the localization of structural genes coding for alloform less cell proteins}

\author{
BY JEAN-LOUIS GUENET, ADRIAN MINTY, BENOIT ROBERT, \\ FRANCOIS BONHOMME AND MARGARET BUCKINGHAM \\ Institut Pasteur, 25 rue du Docteur Roux, 75724 Paris Cedex 15, France
}

The majority of basic cellular proteins and in particular those of the cytoskeleton (vimentin, tubulin, etc.) have extremely invariable primary structures and do not generally exist as isoforms. Mapping of the structural genes concerned can therefore only be achieved by using certain relatively specialized techniques (such as in situ chromosome hybridization) or by a somatic cell genetic approach 
involving the use of molecular probes and interspecific somatic cell hybrids. By analysing the segregation patterns of offspring from crosses involving different mouse species belonging to the Mus genus such as Mus spretus, Mus musculus domesticus and Mus musculus musculus for both DNA polymorphisms as revealed by restriction enzyme site analysis, and segregation of coat colour, immunoglobulin allotypes or other biochemical markers we have been able to map precisely structural genes coding for several such basic cellular proteins. This technique is applicable generally to any situation in which a molecular probe is available.

\title{
Homozygous lethality and Heterozygous recovery in the 'Lethal gray' hamster (Mesocricetus auratus)
}

\author{
BY ALISON J. HOLLOWAY AND JANET E. HORNBY \\ Department of Pure \& Applied Zoology, The University of Reading, \\ Whiteknights, Reading RH6 $2 A J$
}

Lethal gray is an autosomal mutant in the Syrian Hamster. Heterozygotes have a grey coat, homozygotes die in utero (Nixon \& Connelly, Journal of Heredity, 58, 295-296, 1967). A systematic study of uteri and embryos from heterozygote matings has revealed two groups of abnormal embryos. The blastocysts form at $3 \frac{1}{2}$ days p.c. and approximately $25 \%$ are abnormal having a reduced number of inner cell mass (ICM) cells. These embryos implant but show little subsequent growth and proliferation, and are resorbed by day 6 p.c. Of the remaining embryos one third develop normally but two thirds show reduced growth between $5_{2}^{1}$ and $6 \frac{1}{2}$ days p.c., then grow rapidly and gastrulate at $7 \frac{1}{2}$ days, about $24 \mathrm{~h}$ later than the controls. By 10 days p.c. most of these embryos are difficult to distinguish from control embryos. Thus embryos homozygous for Lethal gray form abnormal blastocysts with small ICMs, and die shortly after implantation. Heterozygous embryos are also affected by the Lethal gray gene, forming small abnormal egg cylinders, but are able to recover from the effects of this gene to become viable adults.

\section{Genetic analysis of the mouse $Y$-chromosome}

\author{
BY JAS. K. JUTLEY AND ALISTAIR D. STEWART \\ Department of Chemical Pathology, The Old Medical School, Leeds LS2 9JT
}

$Y$-chromosomal inheritance was demonstrated in the mouse by making use of inbred congenic lines carrying two pairs of $Y$-chromosomes derived from four different inbred strains. Comparisons with one pair of $Y$-chromosomes showed effects on testis weight, sensitivity of certain target organs to exogenous androgen, spermatozoan head abnormality and aggressive behaviour. The second pair of $Y$-chromosomes affected serum testosterone levels, spermatozoan head abnor- 
mality, aggressive and sexual behaviour, and there was preliminary evidence for an effect on male tooth size. No $Y$-chromosome effects were observed on androgendependent target organ weights, sex-ratio of offspring, or on the male-specific antigen (as defined by transplantation or in vitro cell-mediated assays) in these comparisons. Genetic comparisons between these two pairs of $Y$-chromosomes lead to the postulation of a minimum of four loci to explain the observed $Y$-chromosome effects. One locus affects testis weight and sensitivity of target organs to testosterone; one locus affects spermatozoan head abnormality; one locus affects tooth size, serum testosterone levels and sexual behaviour; and one locus affects aggressive behaviour. The characters affected by the same locus may be causally related. For example, testosterone sensitivity could mediate the testis weight effect; androgen levels could mediate the sexual behaviour effect. From the human literature, the tooth size and androgen effects are separate. This suggests a fifth locus. The use of further congenic strains of mice may further sub-divide some of these loci.

\title{
Expression of maternally and paternally derived allozymes of phosphoglycerate kinase 1 (PGK-1) in the early mouse embryo and in the somatic mosaic of the mother
}

\author{
By W. KRIETSCH, R. FUNDELE AND Th. BÜCHER \\ Institute of Physiological Chemistry of the University, Goethestr. 33, \\ D 8000 München 2, Germany
}

Minor modifications of the fluorometric assay for PGK, which has been communicated in earlier meetings, allow for the quantification of allozyme profiles of single eggs and subsequent stages of early development in the mouse. The time course of the reactivation of $P g k-1$ alleles of maternally and paternally derived $X$-chromosomes $(X m$ and $X p$ ) has been studied. Two experimental lines have been followed up. They differed with respect to the maternal genotype. In the first setup, homozygous females were crossed to males carrying the opposite allele. First deviations from the maternal phenotype were observed in the egg cylinders (in 42 of 105 specimens, 6 days p.c.) whereas in 298 specimens, ranging from the egg to the late blastocyst, the allozyme coded by $X p$ was not expressed. In the second setup heterozygous females originating from congenic parents were used. Here significant deviations from the allozyme pattern exhibited by the egg appeared already in the transition from the early to the late blastocyst (day 4 to day 5). They indicate an early expression of the maternally derived $P g k$ allele, the reactivation of the paternally derived locus during preimplantation-stages being excluded by the first set of measurements. It is suggestive to correlate the described phenomenon of 'non-random $X$-chromosome activation' with the simultaneous development of the primitive endoderm and trophectoderm lineages, which will not express the allozyme coded on the paternally derived $X$-chromosome. The inactivation of $X p$ in the course of spermatogenesis might possibly not be reverted in the extra-embryonic lines. In confirmation of previous investigators, both 
allozymes are expressed in the egg cells of heterozygous mothers. However a highly significant preponderance of the wild-type activity (PGK-1B) is observed. Correspondingly in oocytes of homozygous females higher levels of absolute activity of PGK-1B (23.0 $\pm 3.0 ; 77$ oocytes in 14 assays) than of PGK-1A $(11.2 \pm 2.9 ; 82$ in 11) are found. This is in contrast to the expression of the PGK-allozymes in somatic cells. When isolated from such cells PGK-1B and PGK-1A exhibit similar specific activity, are of comparable stability and are expressed at the same level of activity. Moreover in the somatic mosaic of the investigated heterozygotes cells expressing the variant enzyme (PGK-1A) prevail in a proportion of two to one. Imbalanced lyonization is explained by Cattanach by the close association of the Pgk-alleles with different alleles of another locus, called Xce. Experiments with carriers of different alleles of Xce are underway in order to see if correlations exist between the curious findings of PGK-expression in oocytes and the close linkage between the Pgk and Xce.

\title{
Genetic and molecular analysis of the $t$ complex of the mouse
}

\author{
BY H. LEHRACH, BERNHARD HERMANN, HOWARD FOX, \\ DAN RÖHME, JAN-ERIK EDSTRÖM, PAUL MAINS, LEE SILVER \\ AND ANNEMARIE FRISCHAUF \\ European Molecular Biology Laboratory, Postfach 10.2209, 6900 Heidelberg, \\ Federal Republic of Germany
}

Fragments of the proximal half of mouse chromosome 17 were microdissected from metaphase spreads. $D N A$ from 270 chromosome fragments was extracted and cloned into a lambda phage vector using microtechniques. Clones carrying unique or low repetitive sequences were further subcloned and tested for their usability both as genetic markers and as molecular probes to the $t$ complex region. Nine clones defining $t$ complex specific restriction fragment length polymorphisms have been genetically mapped to the $t$ complex, or $t$ complex subregions defined by partial $t$ haplotypes. In addition, cosmid clones have been isolated containing microclone sequences with the aim of covering subregions of the $t$ complex by overlapping cosmid clones.

\section{Further data on the genetic basis of segregation distortion and sterility caused by mouse $t$-haplotypes}

\author{
BY MARY F. LYON \\ MRC Radiobiology Unit, Harwell, Didcot, Oxon, U.K.
}

Previous work has shown that the segregation distortion characteristic of male mice heterozygous for $t$-haplotypes is due to the action of two distorter loci, $T c d-1$ and $T c d-2$, on a responder locus, $T c r-1$, which lies between them. When both 
distorters are present the chromosome carrying the responder is transmitted at high frequency. When the various factors are separated, in partial $t$-haplotypes, the responder is transmitted at a low frequency (ca. $-20 \%$ ). If both distorter loci act in cis or trans, as postulated, a chromosome carrying the responder only should be transmitted at high frequency when placed over one carrying both distorters. This result has now been achieved. The two distorters appear not to be identical, since the proximal distorter $T c d-1$ alone appears not to raise greatly the transmission ratio of the responder, whereas $T c d-2$ alone produces a partial rise in ratio. Similarly, there are at least two loci, located proximally and distally, concerned in male sterility and again they appear not to be identical. The proximal sterility factor does not cause sterility alone but only in combination with the distal factor, whereas homozygosity for the distal factor only is sufficient to cause sterility.

\title{
Expression of $\mathrm{H}-\mathrm{Y}$ antigen by mice carrying $\mathbf{S x r}$
}

\author{
BY ANNE MCLAREN, ELIZABETH SIMPSON*, PHILLIP CHANDLER* \\ AND KYUHEI TOMONARI* \\ MRC Mammalian Development Unit, University College, Wolfson House, \\ 4 Stephenson Way, NW1 2HE *Transplantation Biology Section, \\ Clinical Research Centre, Watford Road, Harrow, \\ Middlesex, HAI $3 U J$
}

The minor transplantation antigen $\mathrm{H}-\mathrm{Y}$ can cause graft rejection and can stimulate the generation of $\mathrm{H}-2$ restricted $\mathrm{T}$ cell responses. We have used both responses to type karyotypically abnormal mice for the presence of $\mathrm{H}-\mathrm{Y}$ antigen, in order to investigate the role of $\mathrm{H}-\mathrm{Y}$ in sex determination. The mice under scrutiny were $S x r$-carrying females derived by crossing females carrying the $T(16 ; X) 16 H$ translocation with $S x r$-carrying males. These females were fully fertile and were H-Y positive. These results are consistent with the testis determining gene, $T d y$, which may or may not be H-Y, having a threshold effect on testis differentiation during embryogenesis. They also show that the presence of $\mathrm{H}-\mathrm{Y}$ in adult females does not impair reproduction.

\section{Ovarian involvement in chromosome anomalies leading to male sterility in the mouse}

\author{
BY URSULA MITTWOCH, SHANTHA MAHADEVAIAH \\ AND LESLIE A. SETTERFIELD \\ Department of Genetics and Biometry, The Galton Laboratory, \\ University College London, Wolfson House, \\ 4 Stephenson Way, London NW1 $2 H E$
}

Ovarian volumes were measured in immature mice with three different chromosome anomalies that lead to male sterility: (1) heterozygous carriers of the 
reciprocal translocation $T(11 ; 19) 42 H$; (2) heterozygous carriers of the insertion $I s(7 ; 1) 40 H ;(3)$ tertiary trisomics $(T s 31 H)$ of $T(5 ; 12) 31 H$. In all three chromosome anomalies, immature females had smaller ovaries than their litter mates with normal chromosomes, suggesting that ovarian involvement is a common phenomenon in male sterility of chromosomal origin. It remains to be investigated whether the smaller ovarian size is associated with a reduction in the number of oocytes. There is some indication that the time at which ovarian size begins to fall behind may differ in the three anomalies.

\title{
Eday mouse versus May mouse: the preliminary results of a field introduction and competition experiment
}

\author{
By H. R. NASH and G. S. TRIGGS \\ Department of Zoology, University College London, Gower Street, \\ London WC1E $6 B T$ \\ Biology Department, Liverpool Polytechnic, Byrom Street, Liverpool
}

The Isle of May in the Firth of Forth, Scotland, supports a flourishing population of feral house-mice (Mus musculus). Of over 80 electrophoretic markers examined only $2, X l d-1$ and $T r f$, have been found to be heterozygous in a total of 7 individuals. The H-2 complex is also polymorphic. The animals have a normal karyotype. In the Spring of 198270 mice from the Orkney island of Eday were released onto the southernmost tip of the island. Eday mice contain a number of useful biochemical markers, a coat colour polymorphism, and have a karyotype of $2 n=34$. Preliminary observations on the effect of this introduction suggest that initially the Eday mice may have out competed the May mice and have hybridized freely with them.

\section{A study of first meiotic metaphase in trisomies 16 and 19 of the female mouse}

\author{
By P. E. POLANI*, J. A. CROLLA* AND A. GROPP** \\ *Paediatric Research Unit, Guy's Hospital Medical School, Guy's Tower, \\ London Bridge SE1 9RT and **Institut für Pathologie der Medizinischen \\ Hochschule Lübeck, West Germany
}

A study of chromosome association at the first meiotic metaphase in trisomies 16 and 19 of the female mouse was carried out by 'rescuing' fetal ovaries from their lethal environment, utilising an in vitro organ culture technique followed by in vivo heterotopic transplantation into spayed female recipients. Murine autosomal trisomy was induced by mating $\mathrm{A} / \mathrm{Strong}$ (all acrocentric) females with males heterozygous for two Robertsonian ( $R b)$ metacentrics with monobrachial homology. Pregnant females were sacrificed on $d 14(\mathrm{plug}=d 1)$, and the products of conception screened morphologically for stigmata of trisomy, and cytogenetically. 
The incidence of the two trisomies, 16 and 19, in the A/Strong background, was $10 \%$ and $15 \%$, respectively. In the successfully transplanted trisomic ovaries, the yield of first meiotic metaphases was much higher in trisomy 19 than in trisomy 16. In trisomy 16 first meiotic metaphases, acrocentric univalents were seen in about $5 \%$ of cells, while in trisomy 19 the proportion was about $30 \%$.

\title{
A new type of 'spot test' for the detection of somatic mutations
}

\author{
By D. A. STEPHENSON and A. G. SEARLE \\ MRC Radiobiology Unit, Harwell, Didcot, Oxon, U.K.
}

Mouse pigmentation is controlled by more than fifty loci. Some affect the distribution of melanocytes (e.g. dominant spotting, $W$; Steel, $S l$; piebeld, $s$; belted, $b t$ ) whilst others modify the type (e.g. agouti, $A$; recessive yellow, $e$ ) or quality (e.g. brown, $b$; beige, $b g$; pallid, $p a$; pink-eyed dilution, $p$ ) of pigment produced. Changes in quality may involve a modification in pigment granule size, shape or degree of melanization. Detection of somatic mutations in animals heterozygous for several recessive coat colour mutants (the mammalian 'spot test') relies upon an alteration in the quality of pigmentation. Providing a sufficient qualitative difference exists between granules produced by the various mutants affecting coat pigmentation it should be possible to detect somatic mutational events automatically by extracting the granules from the hair or retina and discriminating between normal and mutant ones. Methods for extracting granules from pigmented tissue have been developed. The physical dimensions of wild-type and mutant granules were measured with an image analyser. Comparison of these results with those obtained using a Coulter counter will be presented. Evidence for detection of somatic mutations at the cellular level, on the basis of changes in granule size, will be presented in support of this potential alternative method of analysis.

\section{'Male-specific antigens - inducers of mammalian testicular differentiation?'}

\author{
BY ALISTAIR D. STEWART \\ Department of Chemical Pathology, University of Leeds
}

It has been proposed that male-specific antigen ( $\mathrm{H}-\mathrm{Y}$ antigen), whose expression is regulated by the mammalian $Y$-chromosome, is responsible for triggering the differentiation of the indifferent gonad as a testis. This hypothesis is reviewed in the light of the current evidence. 


\title{
Flow cytometric analysis of mouse melanocyte granules for somatic mutation studies
}

\author{
By J. A. STYLES, N. R. PRITCHARD and D. A. STEPHENSON* \\ Imperial Chemical Industries PLC, Central Toxicology Laboratory, Alderley Park, \\ Nr. Macclesfield, Cheshire, U.K., and *MRC Radiobiology Unit, Harwell, \\ Oxon, U.K.
}

The mouse coat spot test for somatic mutations in vivo is gaining acceptance as a method for assessing mutagenic and carcinogenic risk to man from chemical mutagens. Flow cytometric analysis has been used in an attempt to improve the speed and resolving power of a new version of the spot test. Mutant melanocyte granules having altered size and/or morphology were compared with wild type granules. Granules from animals with the following phenotypes were isolated from hair and retinas: $a, A, b, b^{\mathrm{c}}, B^{\mathrm{lt}}, b f, b g, d, e p, l n, p^{\mathrm{un}}$ and $p a$. Suspensions of these particles were subjected to two parameter flow cytometry; the particles were illuminated with $640 \mathrm{~nm}$ light from a $0.8 \mathrm{~mW}$ helium-neon laser and the axial light absorption and narrow angle forward light scatter analysed for each melanocyte granule. Pure samples and mixtures of wild type and mutant granules were analysed. Preliminary results demonstrated that in general it was possible to discriminate the mutant granules from the wild type. Several problems were encountered: (a) additional sample treatment was required to ensure that particles did not aggregate during analysis $(b)$ there was poor correlation between size measurements derived from the flow cytometer and those from microscopic examination for some of the granules (c) some granules were difficult to distinguish from wild type with the parameters used. Other cytometric parameters may be useful in analysing melanocyte granules and these will be discussed.

\section{Investigation of the origin of variability among mouse aggregation chimaeras}

\author{
BY JOHN D. WEST, THEODOR BÜCHER*, INGRID M. LINKE* \\ AND MANFRED DUNNWALD* \\ MRC Radiobiology Unit, Harwell, England and *Institüt für Physiologische \\ Chemie, Universität München, Germany
}

The proportions of the two cell populations in mouse aggregation chimaeras are more variable than in $X$-chromosome inactivation mosaics. Falconer and Avery (J. Embryol. exp. Morph. 43, 195-219, 1978) proposed that much of this variability among chimaeras arises at the segregation of the primitive ectoderm and primitive endoderm lineages in the late blastocyst. Variability among $X$-inactivation mosaics arises by $\boldsymbol{X}$-chromosome inactivation which, in the foetus, is likely to occur after the segregation of these two lineages. We aggregated $\mathrm{C} 3 \mathrm{H} / \mathrm{HeH}$ and 
$\mathrm{C} 3 \mathrm{H} / \mathrm{HeHa}-\mathrm{Pgk}-\mathrm{I}^{\mathrm{a}} / \mathrm{Ws}$ mouse embryos and analysed the proportions of the two cell components in various tissues of the mid-term conceptus using quantitative electrophoresis of phosphoglycerate kinase allozymes. Six foetuses were chimaeric and showed greater variability (range 7-90\% PGK-1B) that a series of thirty mosaic foetuses (range 11-50\% PGK-1B). Another six conceptuses were chimaeric with one cell population being excluded from the foetal lineage either at the segregation between primitive ectoderm and primitive endoderm (5 cases) or within the primitive ectoderm lineage ( 1 case). These observations and correlations between lineages indicate that segregation of cells to primitive ectoderm and primitive endoderm increases the variation among chimaeras but considerable variation exists before this segregation occurs.

\title{
Variation for glucose phosphate isomerase in mouse oocytes
}

\author{
BY JOHN D. WEST AND GRAHAM FISHER \\ MRC Radiobiology Unit, Harwell, Didcot, Oxon OX11 ORD, England
}

The activity of the dimeric enzyme phosphate isomerase (GPI) is regulated in oocytes by a cis-acting temporal gene, now known as Gpi-1t. This gene is closely linked to the structural Gpi-1s gene (Peterson \& Wong, Nature 267, 276-279, 1978; McLaren \& Buehr, Genet. Res. 37, 303-309, 1981). Two variants have been reported previously: $G p i-1 t^{\mathrm{a}}$ (in DBA/2 mice) produces lower levels of oocyte GPI than $G p i-1 t^{\mathrm{b}}$ (in C57BL/6 mice). Quantitative cellulose acetate electrophoresis of GPI from unfertilized eggs produced by $G p i-1 s^{\mathrm{a}} / G p i-1 s^{\mathrm{b}}$ heterozygous females revealed a new high-producing $G p i-1 t$ allele which we designate $G p i-1 t^{\mathrm{c}}$. This allele is present in 101/ $\mathrm{H}$ mice and eggs from $(101 / \mathrm{H} \times \mathrm{C} 57 \mathrm{BL} / 0 \mathrm{la}) \mathrm{F}_{1}$ females produced a skewed allozyme distribution of the order $48 \% \mathrm{~A}: 45 \% \mathrm{AB}: 7 \% \mathrm{~B}$. Tests of tissue specificity and genetic linkage are still in progress. No effect of Gpi-1t has been found in spermatozoa or the few adult somatic tissues examined. No recombinants between $G p i-1 s$ and $G p i-1 t$ were found among $22 G p i-1 s^{\mathrm{a}} / G p i-1 s^{\mathrm{b}}$ females from a $(101 / \mathrm{H} \times \mathrm{JU} / \mathrm{FaCt})+\% \times \mathrm{JU} / \mathrm{FaCt}$ o backeross mating.

\section{Developmental staging of monoclonal antibody markers of mouse spermatogenesis with male sterile mutations}

\author{
BY K. R. WILLISON, I. C. SUMMERHAYES, J. POTTER, P. SMITH, \\ R. K. DUDLEY AND M. F. LYON* \\ Chester Beatty Laboratories, Institute of Cancer Research, Fulham Road, \\ London, SW3 6JB and *MRC Radiobiology Unit, Harwell, \\ Didcot, Oxon
}

Thirteen monoclonal antibodies(T1-T13) which react with antigenic determinants present in adult mouse seminiferous tubules have been prepared (Willison, K. R. 
et al., Cold Spring Harbor Cell Proliferation Series on Teratocarcinoma Stem Cells, vol. $X, 1983$ ). Some of the determinants recognized are localized intracellularly and some extracellularly as judged by indirect immunofluorescence tests on unfixed frozen sections and on live germ cells. We have been interested in developing rapid screening procedures for producing monoclonal antibodies specific to cells at different stages of spermatogenesis and have tested these thirteen antibodies for their ability to bind frozen sections of testes from a variety of male sterile mutants. The mutants screened were $W / W^{\mathrm{v}}, S X R /+, T(X ; 16) 16 H, T f m / Y, T(7 ; 19) 145 H$, $t^{h 45} / t^{h 45}, t^{s 6} / t^{h 18}$. Phenotypically they range from absence of germ cells $\left(W / W^{v}\right.$, $S X R /+)$ through meiotic arrest to non-fertilizing spermatozoa $\left(t^{s 6} / t^{h 18}\right)$. Various antibodies (T5, T8, T9, T12, T13) bind to all the mutants suggesting that the determinants recognized are carried by Sertoli cells. Other antibodies however, only bind to some of the mutants; $T 1$, for example, only binds strongly to the t-haplotype compound sterile showing that the determinant is associated with spermatids and/or spermatozoa. Experiments are now in progress to test stage testis specific cDNA clones which we have recently isolated, in Northern blots with total RNA extracted from the testes of these mutants. 